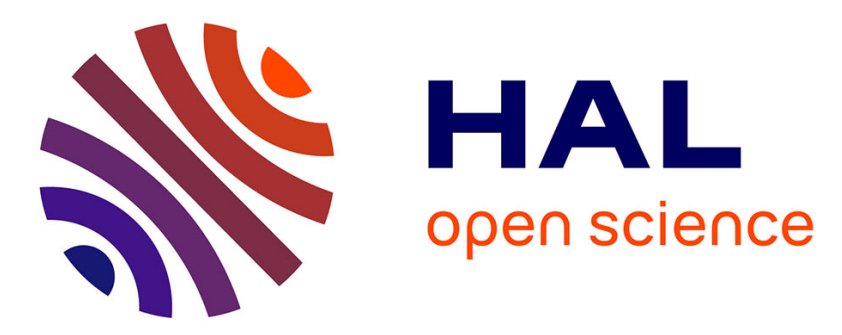

\title{
Jacobi continued fraction and Hankel determinants of the Thue-Morse sequence \\ Guo-Niu Han
}

\section{To cite this version:}

Guo-Niu Han. Jacobi continued fraction and Hankel determinants of the Thue-Morse sequence. Quaestiones Mathematicae, 2016, 39 (7), pp.895-909. 10.2989/16073606.2016.1238855 . hal-02125285

\section{HAL Id: hal-02125285 \\ https://hal.science/hal-02125285}

Submitted on 10 May 2019

HAL is a multi-disciplinary open access archive for the deposit and dissemination of scientific research documents, whether they are published or not. The documents may come from teaching and research institutions in France or abroad, or from public or private research centers.
L'archive ouverte pluridisciplinaire HAL, est destinée au dépôt et à la diffusion de documents scientifiques de niveau recherche, publiés ou non, émanant des établissements d'enseignement et de recherche français ou étrangers, des laboratoires publics ou privés. 
February 5, 2015

\title{
Jacobi continued fraction and Hankel determinants of the Thue-Morse sequence
}

\author{
Guo-Niu Han
}

\begin{abstract}
We study the Jacobi continued fraction and the Hankel determinants of the Thue-Morse sequence and obtain several interesting properties. In particular, a formal power series $\phi(x)$ is being discovered, having the property that the Hankel transforms of $\phi(x)$ and of $\phi\left(x^{2}\right)$ are identical.
\end{abstract}

\section{Introduction}

The Hankel determinants are widely studied in Enumerative Combinatorics [Fl80, Vi83, Kr98, Kr05, La01, Sp06, Pr94], and recently, more attention has been given to the Hankel determinants of the Thue-Morse sequence. As those determinants do not seem to have any closed-form expressions, Modular Arithmetic techniques have been used, as was done by Allouche, Peyrière, Wen and Wen in 1998 [APWW], and in the past year by the author [Ha15, Ha16]. They all proved that such determinants were nonzero, the former by means of a clever determinant manipulation, the latter by using the Jacobi continued fraction expansion. However, Jacobi continued fractions and Hankel determinants, studied from scratch, without using Modular Arithmetic, still have interesting properties. It is the purpose of this paper to discuss some of them.

First, we obtain an explicit expression of the $u$-coefficients in the Jacobi continued fraction expansion of the Thue-Morse sequence, namely, $u_{n}=$ $(-1)^{n+1}$ (see Theorem 2.3 with $b=-1$ ). Second, a curious relation between the generalized Thue-Morse sequence and the Gros sequence [Gr72, HKMP, Go63] is established (Theorem 2.5, Equation (2.12)). Roughly speaking, the Gros sequence can be derived from the generalized ThueMorse sequence by taking the limit as $b$ tends to 1 . Third, we find a formal power series, or an integer sequence, namely,

$$
\begin{aligned}
\phi(x) & =\frac{1}{1+x} \prod_{k=0}^{\infty}\left(1-\left(\frac{x}{1+x}\right)^{2^{k}}\right) \\
& =1-2 x+2 x^{2}-6 x^{4}+20 x^{5}-48 x^{6}+96 x^{7}-166 x^{8}+\cdots
\end{aligned}
$$

Key words and phrases. Hankel determinant, Hankel transform, binomial transform, Jacobi continued fraction, Thue-Morse sequence, Gros sequence.

2010 Mathematics Subject Classification. 05A15, 05A19, 11A55 , 11B37, 11B50, 11B85, 11C20, 15A15. 
which has the property that the Hankel transforms of $\phi(x)$ and of $\phi\left(x^{2}\right)$ are identical (Theorem 2.8). It is worth noting that the sequence $\phi(x)$ does not appear in Sloane's On-Line Encyclopedia of Integer Sequences [S114].

Section 2 is the body of the paper, where we explain the motivation after recalling some basic notations on Jacobi continued fraction and Hankel determinant. Also, the above three results are stated and proved in detail in Section 2, with the help of several useful lemmas described in Section 3.

\section{Motivation, results and open problems}

Let $x$ be an indeterminate. We identify a sequence $\mathbf{a}=\left(a_{0}, a_{1}, a_{2}, \ldots\right)$ with its generating function $f=f(x)=a_{0}+a_{1} x+a_{2} x^{2}+\cdots$. For each $n \geq 1$ and $k \geq 0$ the Hankel determinant of the series $f$ (or of the sequence a) is defined by

$$
H_{n}^{(k)}(f):=\left|\begin{array}{cccc}
a_{k} & a_{k+1} & \ldots & a_{k+n-1} \\
a_{k+1} & a_{k+2} & \ldots & a_{k+n} \\
\vdots & \vdots & \ddots & \vdots \\
a_{k+n-1} & a_{k+n} & \ldots & a_{k+2 n-2}
\end{array}\right|
$$

Let $H_{n}(f):=H_{n}^{(0)}(f)$, for short; the sequence of the Hankel determinants of $f$ is defined to be

$$
H(f):=\left(H_{0}(f)=1, H_{1}(f), H_{2}(f), H_{3}(f), \ldots\right),
$$

and is called the Hankel transform of $f$ (see [La01, SS06]). Let $\mathbf{u}=$ $\left(u_{1}, u_{2}, \ldots\right)$ and $\mathbf{v}=\left(v_{0}, v_{1}, v_{2}, \ldots\right)$ be two sequences. The Jacobi continued fraction attached to $(\mathbf{u}, \mathbf{v})$, or $J$-fraction, for short, is a continued fraction of the form

$$
f(x)=\frac{v_{0}}{1+u_{1} x-\frac{v_{1} x^{2}}{1+u_{2} x-\frac{v_{2} x^{2}}{1+u_{3} x-\frac{v_{3} x^{2}}{\ddots}}}},
$$

also denoted by

$$
f(x)=\mathbf{J}\left[\begin{array}{c}
\mathbf{u} \\
\mathbf{v}
\end{array}\right]=\mathbf{J}\left[\begin{array}{c}
u_{1}, u_{2}, \cdots \\
v_{0}, v_{1}, v_{2}, \cdots
\end{array}\right]
$$

The basic properties on $J$-fractions can be found in [Fl80, Wa48, Vi83, $\mathrm{Kr} 98, \mathrm{Kr} 05]$. The $J$-fraction expansion of a given power series $f(x)$ exists if and only if all the Hankel determinants $H_{n}(f(x))$ of $f(x)$ are nonzero. 
Let $u_{n}(f(x))$ and $v_{n}(f(x))$ denote the coefficients $u_{n}$ and $v_{n}$ in the above $J$-fraction expansion of $f(x)$. Hankel determinants can be calculated from the $J$-fraction by means of the following fundamental relation:

$$
H_{n}\left(\mathbf{J}\left[\begin{array}{c}
u_{1}, u_{2}, \cdots \\
v_{0}, v_{1}, v_{2}, \cdots
\end{array}\right]\right)=v_{0}^{n} v_{1}^{n-1} v_{2}^{n-2} \cdots v_{n-2}^{2} v_{n-1} .
$$

Conversely, the coefficients $u_{n}$ and $v_{n}$ in the $J$-fraction can be calculated from the Hankel determinants by means of the following relations, when all denominators are nonzero:

$$
\begin{aligned}
& u_{n}=-\frac{1}{H_{n-1}^{(1)}}\left(\frac{H_{n-1} H_{n}^{(1)}}{H_{n}}+\frac{H_{n} H_{n-2}^{(1)}}{H_{n-1}}\right), \quad(n \geq 2) \\
& v_{n}=\frac{H_{n} H_{n-2}}{\left(H_{n-1}\right)^{2}} . \quad(n \geq 2)
\end{aligned}
$$

Our motivation was to use the $J$-fraction approach to re-prove the following result obtained by Allouche, Peyrière, Wen and Wen in 1998 [APWW]. Their original proof is based on determinant manipulation, which consists of proving sixteen recurrence relations between determinants.

Theorem 2.1 [APWW]. Let $P_{2}(x)=\prod_{k=0}^{\infty}\left(1-x^{2^{k}}\right)$ be the generating function of the Thue-Morse sequence. Then, $H_{n}\left(P_{2}\right) / 2^{n-1} \equiv 1 \quad(\bmod 2)$ for every positive integer $n$.

Unfortunately, the $v$-coefficients in the $J$-fraction of $P_{2}(x)$ do not have a closed-form expression, as shown by the following first values:

$$
P_{2}(x)=\mathbf{J}\left[\begin{array}{l}
\mathbf{u} \\
\mathbf{v}
\end{array}\right]=\mathbf{J}\left[\begin{array}{c}
1,-1,1,-1,1,-1,1,-1,1,-1,1,-1, \cdots \\
1,-2,1,-1,-1,-1,1,-1,1,-3, \frac{1}{3},-\frac{1}{3},-3, \cdots
\end{array}\right] .
$$

Let $\xi$ be an irrational, real number. The irrationality exponent $\mu(\xi)$ of $\xi$ is the supremum of the real numbers $\mu$ such that the inequality

$$
\left|\xi-\frac{p}{q}\right|<\frac{1}{q^{\mu}}
$$

has infinitely many solutions in rational numbers $p / q$. With the help of Theorem 2.1 Bugeaud [Bu11] was able to prove that the irrationality exponent of the Thue-Morse-Mahler number is equal to 2. On the other hand, Coons [Co13], using the method described in [APWW], proved the following theorem, which is essentially equivalent to another result of Allouche et al., as shown in [BH14].

Theorem 2.2. Let

$$
S_{2}=S_{2}(x)=\frac{1}{x} \sum_{n=0}^{\infty} \frac{x^{2^{n}}}{1-x^{2^{n}}}
$$

be the Gros sequence. Then $H_{n}\left(S_{2}\right) \equiv 1 \quad(\bmod 2)$. 
Again, the coefficients in the $J$-fraction of $S_{2}(x)$ do not have a closedform expression. Thus, we cannot prove Theorem 2.1 using formula (2.3) only, because the coefficients $\left(v_{n}\right)_{n \geq 0}$ are unknown. An appropriate application of Modular Arithmetic provides a bypass and then a solution, as was done in [Ha15, Ha16]. However, we observe that the top coefficients $\left(u_{n}\right)_{n \geq 1}=(1,-1,1,-1,1,-1, \ldots)$ seem to be very simple. This alternance of +1 and -1 can be proved. In fact we have the following general result.

Theorem 2.3. Let $P_{2}(x ; b)$ and its $J$-fraction expansion be

$$
P_{2}(x ; b)=\prod_{k \geq 0}\left(1+b x^{2^{k}}\right)=\mathbf{J}\left[\begin{array}{c}
u_{1}, u_{2}, \cdots \\
v_{0}, v_{1}, v_{2}, \cdots
\end{array}\right] .
$$

Then, $u_{n}=(-1)^{n} b$.

The first values of the $J$-fraction expansion of the above power series $P_{2}(x ; b)$ are

$$
\mathbf{J}\left[\begin{array}{c}
-b, b,-b, b,-b, b,-b, b,-b, b, \cdots \\
1, b-b^{2}, 1,-\left(1+b+b^{2}\right), \frac{-1}{1+b+b^{2}}, \frac{1+b-b^{4}}{1+b+b^{2}},-\frac{\left(1+b+b^{2}\right)^{2}}{1+b-b^{4}}, \cdots
\end{array}\right] .
$$

The structure of $J$-fraction imposes an induction method for finding and proving the $J$-fraction expansion of a power series. For example, we can easily obtain an explicit expression for a periodic $J$-fraction. Since the $v$-coefficients in (2.8) are not periodic, the most difficult part for proving Theorem 2.3 was to find an appropriate generalization required by the induction method. Actually, Theorem 2.3 will appear to be a corollary of Lemma 3.1, whose proof is given in Section 3.

By Theorem 2.3 and formula (2.4), we get

Corollary 2.4. The Hankel determinants $H\left(P_{2}\right)$ and $H^{(1)}\left(P_{2}\right)$ of the Thue-Morse sequence satisfy the following relation (the parameter $\left(P_{2}\right)$ is not reproduced):

$$
H_{n-1}^{2} H_{n}^{(1)}+H_{n}^{2} H_{n-2}^{(1)}=(-1)^{n} H_{n-1}^{(1)} H_{n} H_{n-1} . \quad(n \geq 2)
$$

Consider some special cases of Theorem 2.3. The case $b=0$ is trivial. We get the Thue-Morse sequence by taking $b=-1$. Surprisingly enough, Theorem 2.3 is already proven for $b=I$ by Bacher, where $I$ is the imaginary unit [B06a, B06b]. The case $b=1$ of Theorem 2.3 is especially interesting, and deserves a further discussion. The left-hand side of (2.7) then becomes

$$
\prod_{k \geq 0}\left(1+x^{2^{k}}\right)=\frac{1}{1-x}
$$

and the $J$-fraction is trivial. However, the limit of (2.8) still has a nontrivial meaning as $b$ tends to 1 . We obtain a curious relation between the (generalized) Thue-Morse sequence $P_{2}(x ; b)$ and the Gros sequence $S_{2}(x)$ studied by Coons [Go63, Co13, Gr72, HKMP]. 
Theorem 2.5. Let $n \geq 1$. The Hankel determinant $H_{n}\left(P_{2}(x ; b)\right)$ is divisible by $(1-b)^{n-1}$, and

$$
\lim _{b \rightarrow 1} \frac{H_{n}\left(P_{2}(x ; b)\right)}{(1-b)^{n-1}}=H_{n-1}\left((1-x) S_{2}\left(x^{2}\right)\right) .
$$

Proof. Let

$$
P_{2}(x ; b)=\frac{1}{1-b x+G(x ; b)}
$$

or

$$
G(x ; b):=\frac{1}{\prod_{k \geq 0}\left(1+b x^{2^{k}}\right)}-(1-b x) .
$$

It is easy to verify (1) $G(x, 0)=0 ;(2) G(x, 1)=0 ;(3) G(x, b)=x^{2} \times \cdots$. Thus, $G(x ; b)$ has the following form:

$$
G(x ; b)=b(b-1) x^{2} g(x ; b) .
$$

By (2.10) and (2.7) we have the $J$-fraction expansion for $g(x ; b)$

$$
g(x ; b)=\mathbf{J}\left[\begin{array}{c}
u_{2}, u_{3}, \cdots \\
1, v_{2}, v_{3}, \cdots
\end{array}\right] .
$$

Equation (2.11) is not trivial when $b=1$. Let us evaluate the left-hand side:

$$
\begin{aligned}
g(x ; 1) & =\left.\frac{G(x, b)}{b(b-1) x^{2}}\right|_{b=1}=\left.\frac{G_{b}^{\prime}(x, b)}{x^{2}}\right|_{b=1} \\
& =\frac{1}{x^{2}}\left(x-(1-x) \sum_{k \geq 0} \frac{x^{2^{k}}}{1+x^{2^{k}}}\right) \\
& =\frac{1-x}{x^{2}} \sum_{k \geq 1} \frac{x^{2^{k}}}{1-x^{2^{k}}}=(1-x) S_{2}\left(x^{2}\right),
\end{aligned}
$$

where $S_{2}(x)$ is defined in (2.6). Note that the denominator is changed into $1-x^{2^{k}}$ in the last equality, which can be derived by means of the following identities:

$$
\frac{x}{1-x}=\sum_{n \geq 1} x^{n}=\sum_{k, m \geq 0} x^{(2 m+1) 2^{k}}=\sum_{k \geq 0} x^{2^{k}} \sum_{m \geq 0} x^{m 2^{k+1}}=\sum_{k \geq 0} \frac{x^{2^{k}}}{1-x^{2^{k+1}}}
$$

and

$$
\frac{2 x^{2}}{1-x^{2}}=\sum_{k \geq 1} \frac{2 x^{2^{k}}}{1-x^{2^{k+1}}}=\sum_{k \geq 1} \frac{x^{2^{k}}}{1+x^{2^{k}}}+\sum_{k \geq 1} \frac{x^{2^{k}}}{1-x^{2^{k}}} .
$$


We have

$$
v_{1}\left(P_{2}(x ; b)\right)=(1-b) b,
$$

and, for each $n \geq 1$,

$$
\lim _{b \rightarrow 1} v_{n+1}\left(P_{2}(x ; b)\right)=v_{n}(g(x ; 1))=v_{n}\left((1-x) S_{2}\left(x^{2}\right)\right)
$$

by the identity $(2.12)$. Hence, the Hankel determinant $H_{n}\left(P_{2}(x ; b)\right)$ is divisible by $(1-b)^{n-1}$, and

$$
\lim _{b \rightarrow 1} \frac{H_{n}\left(P_{2}(x ; b)\right)}{(1-b)^{n-1}}=H_{n-1}\left((1-x) S_{2}\left(x^{2}\right)\right) .
$$

This achieves the proof.

Theorem 2.6. We have $H_{n}\left((1-x) S_{2}\left(x^{2}\right)\right) \equiv 1 \quad(\bmod 2)$.

Proof. We make use of the fractional congruence method developed in [Ha15, Section 2]. Let

$$
g(x)=\frac{1-x}{x^{2}} \sum_{k \geq 1} \frac{x^{2^{k}}}{1-x^{2^{k}}}=(1-x) S_{2}\left(x^{2}\right) .
$$

Then,

$$
\frac{x^{2} g(x)}{1-x}=\sum_{k \geq 1} \frac{x^{2^{k}}}{1-x^{2^{k}}}=\frac{x^{2}}{1-x^{2}}+\sum_{k \geq 2} \frac{x^{2^{k}}}{1-x^{2^{k}}}=\frac{x^{2}}{1-x^{2}}+\frac{x^{4} g\left(x^{2}\right)}{1-x^{2}}
$$

or

$$
(1+x) g(x)=1+x^{2} g\left(x^{2}\right) .
$$

Since $g\left(x^{2}\right) \equiv g(x)^{2} \quad(\bmod 2)$ (see, for example, [Ha15, Lemma 2.1]), we have

$$
(1+x) g(x) \equiv 1+x^{2} g(x)^{2} \quad(\bmod 2) .
$$

Hence

$$
g(x) \equiv \frac{1}{1+x-x^{2} g(x)} \equiv \mathbf{J}\left[\begin{array}{l}
(1)^{*} \\
(1)^{*}
\end{array}\right]
$$

where $(1)^{*}$ designates the sequence $(1,1,1, \ldots)$ and the symbol " $\equiv$ " the fractional congruence. By [Ha15, Lemma 2.2(3)] we derive $H_{n}(g(x)) \equiv 1$ $(\bmod 2)$.

By Theorems 2.1, 2.5 and 2.6 we obtain the following results about the $v$-coefficients in the Jacobi continued fraction expansion of the generalized Thue-Morse sequence $P_{2}(x ; b)$. 
Theorem 2.7. Let $v_{n}\left(P_{2}(x ; b)\right)$ be the $v$-coefficients in the Jacobi continued fraction expansion of $P_{2}(x ; b)$ (see (2.8) for the first values). For each $n \geq 2$ the fraction $v_{n}\left(P_{2}(x ; b)\right)$ in the variable $b$ has neither the zero \pm 1 , nor the pole \pm 1 . Moreover,

$$
v_{n}\left(P_{2}(x ; \pm 1)\right) \equiv 1 \quad(\bmod 2) .
$$

Remark. For each polynomial $P(b)$ we have $P(1) \equiv P(-1) \quad(\bmod 2)$. However for a fraction $f(b)$ in the variable $b$ the statement " $f(1) \equiv f(-1)$ $(\bmod 2) "$ is not true in general. For example, taking

$$
f(b)=\frac{1+b^{2}}{1+b+b^{2}+b^{4}},
$$

we have $f(-1) \equiv 1 \quad(\bmod 2)$. However $f(1)=1 / 2 \not \equiv 1 \quad(\bmod 2)$. Hence the cases $b=+1$ and $b=-1$ are independent in Theorem 2.7.

In [B06a, B06b] Bacher proved an iterative formula for the Hankel determinants of the power series

$$
\prod_{k \geq 0}\left(1+I x^{2^{k}}\right)
$$

A similar result has been recently established by $\mathrm{Wu}$ and the author [HW15] for the power series

$$
\prod_{k \geq 0}\left(1+J x^{3^{k}}\right)
$$

where $J=(I \sqrt{3}-1) / 2$.

The study of the Hankel determinants of the Thue-Morse sequence led us to derive a power series $F(x)$ such that the Hankel transforms of $F(x)$ and of $F\left(x^{2}\right)$ are identical.

Theorem 2.8. Let

$$
\Phi(x)=\frac{1}{1+b^{2} x} \prod_{k=0}^{\infty}\left(1+b\left(\frac{x}{1+b^{2} x}\right)^{2^{k}}\right) .
$$

Then, the two series $\Phi(x)$ and $\Phi\left(x^{2}\right)$ have the same Hankel transform, i.e. $H_{n}(\Phi(x))=H_{n}\left(\Phi\left(x^{2}\right)\right)$ for all positive integers $n$.

Proof. Theorem 2.8 is a consequence of Lemma 3.7 by taking the special values $c:=b^{2}$ and $F(x):=P_{2}(x ; b)$. Since $P_{2}(x ; b)=(1+b x) P_{2}\left(x^{2} ; b\right)$, 
we have $G(x)=(1+b x) F\left(x^{2}\right)=F(x)$ in Lemma 3.7. Hence, the Hankel transforms of $\bar{G}(x)$ and $\tilde{G}(x)=\bar{G}\left(x^{2}\right)$ are identical. The proof is achieved by the following calculation:

$$
\begin{aligned}
\bar{G}(x) & =\frac{1}{1+c x} G\left(\frac{x}{1+c x}\right) \\
& =\frac{1}{1+b^{2} x} F\left(\frac{x}{1+b^{2} x}\right) \\
& =\frac{1}{1+b^{2} x} P_{2}\left(\frac{x}{1+b^{2} x} ; b\right)=\Phi(x) .
\end{aligned}
$$

The coefficients of the power series $\Phi(x)=\sum_{n \geq 0} d_{n} x^{n}$ can be explicitly expressed as follows:

$$
d_{n}=\sum_{k=0}^{n}(-1)^{n-k}\left(\begin{array}{l}
n \\
k
\end{array}\right) b^{2 n-2 k+\beta(k)},
$$

where $\beta(k)$ is the number of 1 's of the integer $k$ in the binary numeral system. The first terms of $\Phi(x)$ are

$$
\begin{aligned}
\Phi(x)=1 & +\left(-b^{2}+b\right) x+\left(b^{4}-2 b^{3}+b\right) x^{2}+\left(-b^{6}+3 b^{5}-3 b^{3}+b^{2}\right) x^{3} \\
& +\left(b^{8}-4 b^{7}+6 b^{5}-4 b^{4}+b\right) x^{4}+\cdots,
\end{aligned}
$$

whose specialization for $b=-1$ reads

$\phi(x)=1-2 x+2 x^{2}-6 x^{4}+20 x^{5}-48 x^{6}+96 x^{7}-166 x^{8}+252 x^{9}+\cdots$

We end the section by stating some conjectures and problems. The following conjecture generalizes Theorem 2.7.

Conjecture 2.9. Let $v_{n}\left(P_{2}(x ; b)\right)$ be the $v$-coefficients in the Jacobi continued fraction expansion of $P_{2}(x ; b)$ (see (2.8) for the first values). For each $n \geq 2$ the fraction $v_{n}\left(P_{2}(x ; b)\right)$ in the variable $b$ contains only factors $\Gamma(b)$ such that $\Gamma(1)$ is odd.

For example, the fraction $v_{14}\left(P_{2}(x ; b)\right.$ is equal to

$$
\frac{\left(b^{6}+b^{5}+b^{4}+b^{3}+b^{2}+b+1\right)\left(b^{8}-b-1\right)}{b^{14}-b^{13}-b^{11}-b^{10}-b^{8}-b^{7}+b^{5}+2 b^{3}+b+1}
$$

which contains three factors

$$
\begin{aligned}
& \Gamma_{1}(b)=b^{6}+b^{5}+b^{4}+b^{3}+b^{2}+b+1 ; \\
& \Gamma_{2}(b)=b^{8}-b-1 ; \\
& \Gamma_{3}(b)=b^{14}-b^{13}-b^{11}-b^{10}-b^{8}-b^{7}+b^{5}+2 b^{3}+b+1
\end{aligned}
$$

such that $\Gamma_{1}(1), \Gamma_{2}(1), \Gamma_{3}(1)$ are all odd integers. 
Problem 2.10. Characterize all the formal power series $f(x)$ such that $f(x)$ and $f\left(x^{2}\right)$ have the same Hankel transform.

Problem 2.11. Find a nice formal power series $f(x)$ such that $f(x)$ and $f\left(x^{3}\right)$ have the same Hankel transform.

\section{Properties on $J$-fraction and Hankel determinant}

In this section we establish several properties on Jacobi continued fractions and Hankel determinants. These properties are useful for proving the theorems stated in Secion 2. In particulier, the following Lemma generalizes Theorem 2.3 and is easy to prove by using the iteration method. However Theorem 2.3 has remained unsolved for a longtime, until Lemma 3.1 was discovered.

Lemma 3.1. Let $b$ be an indeterminate and $F(x)$ be a formal power series. Define

$$
G(x):=(1+b x) F\left(x^{2}\right) .
$$

If the Jacobi fraction expansion of $G(x)$ exists, then

$$
G(x)=\mathbf{J}\left[\begin{array}{c}
u_{1}, u_{2}, \cdots \\
v_{0}, v_{1}, v_{2}, \cdots
\end{array}\right]=\mathbf{J}\left[\begin{array}{c}
-b, b,-b, b, \cdots \\
v_{0}, v_{1}, v_{2}, v_{3}, v_{4}, \cdots
\end{array}\right] .
$$

In other words, $u_{n}=(-1)^{n} b$.

Proof. We can define $v_{0}$ and $G_{1}(x)$ by the condition

$$
G(x)=\frac{v_{0}}{1-b x+G_{1}(x) x^{2}} .
$$

Since the $J$-fraction of $G(x)$ exists, we have $G(0) \neq 0$. Let $v_{0}=G(0)=$ $F(0) \neq 0$. Condition (3.3) is equivalent to

$$
\begin{aligned}
G_{1}(x) x^{2} & =\frac{v_{0}}{G(x)}-(1-b x) \\
& =\frac{v_{0}}{(1+b x) F\left(x^{2}\right)}-(1-b x) \\
& =\frac{v_{0}(1-b x)}{\left(1-b^{2} x^{2}\right) F\left(x^{2}\right)}-(1-b x) \\
& =(1-b x)\left(\frac{v_{0}}{\left(1-b^{2} x^{2}\right) F\left(x^{2}\right)}-1\right) .
\end{aligned}
$$

Hence,

$$
G_{1}(x)=(1-b x) \times \frac{1}{x^{2}}\left(\frac{v_{0}}{\left(1-b^{2} x^{2}\right) F\left(x^{2}\right)}-1\right) .
$$


Again, the power series $G_{1}(x)$ is the product of $(1-b x)$ and an even series. By comparing (3.1) and (3.4), we can define $v_{1}$ and $G_{2}(x)$ by the condition

$$
G_{1}(x)=\frac{v_{1}}{1+b x+G_{2}(x) x^{2}} .
$$

In the same manner, $G_{2}(x)$ is the product of $(1+b x)$ and an even series. We obtain equality (3.2) by iteration.

Layman [La01] proved that the Hankel transform is invariant under the binomial transform. Spivey et al. [SS06] proved that the Hankel transform is invariant under the falling $k$-binomial transform, which maps the sequence $\left(a_{n}\right)$ to $\left(a_{n}^{\prime}\right)$ defined by

$$
a_{n}^{\prime}:=\sum_{k=0}^{n}\left(\begin{array}{l}
n \\
k
\end{array}\right)(-b)^{n-k} a_{k} .
$$

The generating function for the sequence $\left(a_{n}^{\prime}\right)$ is equal to

$$
\frac{1}{1+b x} F\left(\frac{x}{1+b x}\right)
$$

where $F(x)$ is the generating function for the sequence $\left(a_{n}\right)$. See $[\operatorname{Pr} 94$, $\mathrm{Ba} 09]$.

Lemma 3.2. We have

$$
H_{n}^{(k)}(F(x))=H_{n}^{(k)}\left((1+b x)^{k-1} F\left(\frac{x}{1+b x}\right)\right) .
$$

Proof. The case $k=0$ is just the result by Spivey et al. and Prodinger mentioned above. If $k \geq 1$, then $H_{n}^{(k)}(F(x))=H_{n}^{(0)}(f(x))$ where

$$
\begin{aligned}
F(x) & =a_{0}+a_{1} x+a_{2} x^{2}+\cdots+a_{k-1} x^{k-1}+a_{k} x^{k}+\cdots, \\
p(x) & =a_{0}+a_{1} x+a_{2} x^{2}+\cdots+a_{k-1} x^{k-1} \\
f(x) & =a_{k-1}+a_{k} x+\cdots, \\
F(x) & =p(x)+x^{k} f(x) \\
f(x) & =\frac{F(x)-p(x)}{x^{k}}
\end{aligned}
$$

Setting $k=0$ and $F=f$ in Identity (3.5) gives

$$
H_{n}^{(0)}(f(x))=H_{n}^{(0)}\left(\frac{1}{1+b x} f\left(\frac{x}{1+b x}\right)\right) .
$$


On the other hand,

$$
(1+b x)^{k-1} F\left(\frac{x}{1+b x}\right)=(1+b x)^{k-1} p\left(\frac{x}{1+b x}\right)+x^{k} \frac{1}{1+b x} f\left(\frac{x}{1+b x}\right),
$$

and $(1+b x)^{k-1} p\left(\frac{x}{1+b x}\right)$ is a polynomial in $x$ of degree $k-1$. Consequently,

$$
H_{n}^{(0)}\left(\frac{1}{1+b x} f\left(\frac{x}{1+b x}\right)\right)=H_{n}^{(k)}\left((1+b x)^{k-1} F\left(\frac{x}{1+b x}\right)\right) .
$$

By (3.6) and (3.7), Equality (3.5) holds.

The following two identities about Hankel determinants are well-known (see, for example, [GX06, Kr05])

$$
\begin{gathered}
H_{2 n}\left(F\left(x^{2}\right)\right)=H_{n}(F(x)) \times H_{n}^{(1)}(F(x)), \\
H_{2 n+1}\left(F\left(x^{2}\right)\right)=H_{n+1}(F(x)) \times H_{n}^{(1)}(F(x)),
\end{gathered}
$$

and can be generalized as stated next.

Lemma 3.3. Let $b$ be a parameter and $F(x)$ be a formal power series. Then,

$$
\begin{aligned}
H_{2 n}\left((1+b x) F\left(x^{2}\right)\right) & =H_{n}(F(x)) \times H_{n}^{(1)}\left(\left(1-b^{2} x\right) F(x)\right), \\
H_{2 n+1}\left((1+b x) F\left(x^{2}\right)\right) & =H_{n+1}(F(x)) \times H_{n}^{(1)}\left(\left(1-b^{2} x\right) F(x)\right) .
\end{aligned}
$$

Proof. Without loss of generality, we illustrate the evaluation of the Hankel determinant (3.10) for $n=3$. Let $F(x)=\sum_{n \geq 0} c_{n} x^{n}$. Then

$$
(1+b x) F\left(x^{2}\right)=c_{0}+b c_{0} x+c_{1} x^{2}+b c_{1} x^{3}+\cdots
$$

By permuting rows and columns, we have

$$
\begin{aligned}
H_{2 n}\left((1+b x) F\left(x^{2}\right)\right) & =(-1)^{3}\left|\begin{array}{cccccc}
c_{0} & b c_{0} & c_{1} & b c_{1} & c_{2} & b c_{2} \\
c_{1} & b c_{1} & c_{2} & b c_{2} & c_{3} & b c_{3} \\
c_{2} & b c_{2} & c_{3} & b c_{3} & c_{4} & b c_{4} \\
b c_{0} & c_{1} & b c_{1} & c_{2} & b c_{2} & c_{3} \\
b c_{1} & c_{2} & b c_{2} & c_{3} & b c_{3} & c_{4} \\
b c_{2} & c_{3} & b c_{3} & c_{4} & b c_{4} & c_{5}
\end{array}\right| \\
& =(-1)^{6}\left|\begin{array}{cccccc}
c_{0} & c_{1} & c_{2} & b c_{0} & b c_{1} & b c_{2} \\
c_{1} & c_{2} & c_{3} & b c_{1} & b c_{2} & b c_{3} \\
c_{2} & c_{3} & c_{4} & b c_{2} & b c_{3} & b c_{4} \\
b c_{0} & b c_{1} & b c_{2} & c_{1} & c_{2} & c_{3} \\
b c_{1} & b c_{2} & b c_{3} & c_{2} & c_{3} & c_{4} \\
b c_{2} & b c_{3} & b c_{4} & c_{3} & c_{4} & c_{5}
\end{array}\right|
\end{aligned}
$$




$$
\begin{aligned}
& =\left|\begin{array}{cccccc}
c_{0} & c_{1} & c_{2} & b c_{0} & b c_{1} & b c_{2} \\
c_{1} & c_{2} & c_{3} & b c_{1} & b c_{2} & b c_{3} \\
c_{2} & c_{3} & c_{4} & b c_{2} & b c_{3} & b c_{4} \\
0 & 0 & 0 & c_{1}-b^{2} c_{0} & c_{2}-b^{2} c_{1} & c_{3}-b^{2} c_{2} \\
0 & 0 & 0 & c_{2}-b^{2} c_{1} & c_{3}-b^{2} c_{2} & c_{4}-b^{2} c_{3} \\
0 & 0 & 0 & c_{3}-b^{2} c_{2} & c_{4}-b^{2} c_{3} & c_{5}-b^{2} c_{4}
\end{array}\right| \\
= & H_{n}(F) H_{n}(g),
\end{aligned}
$$

where

$$
\begin{aligned}
g(x) & =\left(c_{1}-b^{2} c_{0}\right)+\left(c_{2}-b^{2} c_{1}\right) x+\left(c_{3}-b^{2} c_{2}\right) x^{2}+\cdots \\
& =\frac{1}{x}\left(\left(1-b^{2} x\right) F(x)-c_{0}\right) .
\end{aligned}
$$

Equality $(3.10)$ is proved since $H_{n}(g)=H_{n}^{(1)}\left(\left(1-b^{2} x\right) F(x)\right)$. In the same manner, the Hankel determinant (3.11) is evaluated as follows.

$$
\begin{aligned}
H_{2 n+1}\left((1+b x) F\left(x^{2}\right)\right) & =(-1)^{3}\left|\begin{array}{ccccc}
c_{0} & b c_{0} & c_{1} & b c_{1} & c_{2} \\
c_{1} & b c_{1} & c_{2} & b c_{2} & c_{3} \\
c_{2} & b c_{2} & c_{3} & b c_{3} & c_{4} \\
b c_{0} & c_{1} & b c_{1} & c_{2} & b c_{2} \\
b c_{1} & c_{2} & b c_{2} & c_{3} & b c_{3}
\end{array}\right| \\
& =(-1)^{6}\left|\begin{array}{ccccc}
c_{0} & c_{1} & c_{2} & b c_{0} & b c_{1} \\
c_{1} & c_{2} & c_{3} & b c_{1} & b c_{2} \\
c_{2} & c_{3} & c_{4} & b c_{2} & b c_{3} \\
b c_{0} & b c_{1} & b c_{2} & c_{1} & c_{2} \\
b c_{1} & b c_{2} & b c_{3} & c_{2} & c_{3}
\end{array}\right| \\
& =\left|\begin{array}{llllll}
c_{0} & c_{1} & c_{2} & b c_{0} & b c_{1} \\
c_{1} & c_{2} & c_{3} & b c_{1} & b c_{2} \\
c_{2} & c_{3} & c_{4} & b c_{2} & b c_{3} \\
0 & 0 & 0 & c_{1}-b^{2} c_{0} & c_{2}-b^{2} c_{1} \\
0 & 0 & 0 & c_{2}-b^{2} c_{1} & c_{3}-b^{2} c_{2}
\end{array}\right| \\
& =H_{n+1}(F) H_{n}(g) \\
& =H_{n+1}(F) H_{n}^{(1)}\left(\left(1-b^{2} x\right) F(x)\right) .
\end{aligned}
$$

The following two Lemmas can be proved in the same way by using determinant manipulations.

Lemma 3.4. Let $b$ be a parameter and $F(x)$ be a formal power series. We have

$$
\begin{aligned}
H_{3 n}(G) & =(-1)^{n} H_{n}(F) H_{n}^{(1)}(F) H_{n}^{(1)}(g), \\
H_{3 n+1}(G) & =(-1)^{n} H_{n+1}(F) H_{n}^{(1)}(F) H_{n}^{(1)}(g), \\
H_{3 n+2}(G) & =(-1)^{n+1} b^{2}\left(H_{n+1}(F)\right)^{2} H_{n}^{(2)}(g),
\end{aligned}
$$

where $G(x):=(1+b x) F\left(x^{3}\right)$ and $g(x):=\left(1+b^{3} x\right) F(x)$. 
Lemma 3.5. Let $b$ be a parameter and $F(x)$ be a formal power series. We have

$$
H_{3 n+2}\left(\left(1+b x^{2}\right) F\left(x^{3}\right)\right)=(-1)^{n} b\left(H_{n+1}(F)\right)^{2} H_{n}^{(2)}\left(\left(1+b^{3} x^{2}\right) F(x)\right) .
$$

Remark. Unlike Lemma 3.4, there is no analogous factorization formula for $H_{3 n}\left(\left(1+b x^{2}\right) F\left(x^{3}\right)\right)$ and $H_{3 n+1}\left(\left(1+b x^{2}\right) F\left(x^{3}\right)\right)$. When $b=0$, Lemma 3.4 becomes the following corollary.

Corollary 3.6. Let $F(x)$ be a formal power series. We have

$$
\begin{aligned}
H_{3 n}\left(F\left(x^{3}\right)\right) & =(-1)^{n} H_{n}(F)\left(H_{n}^{(1)}(F)\right)^{2}, \\
H_{3 n+1}\left(F\left(x^{3}\right)\right) & =(-1)^{n} H_{n+1}(F)\left(H_{n}^{(1)}(F)\right)^{2}, \\
H_{3 n+2}\left(F\left(x^{3}\right)\right) & =0 .
\end{aligned}
$$

Lemma 3.7. Let $b, c$ be two parameters and $F(x)$ be a formal power series. Define another three power series by

$$
\begin{aligned}
G(x) & :=(1+b x) F\left(x^{2}\right), \\
\bar{G}(x) & :=\frac{1}{1+c x} G\left(\frac{x}{1+c x}\right), \\
\tilde{G}(x) & :=\frac{1}{1+b^{2} x^{2}} F\left(\frac{x^{2}}{1+b^{2} x^{2}}\right) .
\end{aligned}
$$

Then, the Hankel determinants of $G, \bar{G}$ and $\tilde{G}$ are equal. In other words,

$$
H_{n}(G)=H_{n}(\bar{G})=H_{n}(\tilde{G}) \quad(n \geq 0) .
$$

Proof. By Lemma 3.2 we have $H_{n}(G)=H_{n}(\bar{G})$. Let

$$
f(x)=\frac{1}{1+b^{2} x} F\left(\frac{x}{1+b^{2} x}\right)
$$

such that $\tilde{G}(x)=f\left(x^{2}\right)$. By Lemma 3.2, $H_{n}(f(x))=H_{n}(F(x))$. By $(3.8-3.9)$

$$
\begin{aligned}
H_{2 n}(\tilde{G}) & =H_{n}(f) H_{n}^{(1)}(f)=H_{n}(F) H_{n}^{(1)}(f), \\
H_{2 n+1}(\tilde{G}) & =H_{n+1}(f) H_{n}^{(1)}(f)=H_{n+1}(F) H_{n}^{(1)}(f) .
\end{aligned}
$$

By Lemmas 3.3 and 3.2 with $k=1$

$$
\begin{aligned}
H_{2 n}(G) & =H_{n}(F) H_{n}^{(1)}\left(\left(1-b^{2} x\right) F(x)\right) \\
& =H_{n}(F) H_{n}^{(1)}\left(\left(1-b^{2} \frac{x}{1+b^{2} x}\right) F\left(\frac{x}{1+b^{2} x}\right)\right) \\
& =H_{n}(F) H_{n}^{(1)}(f(x))=H_{2 n}(\tilde{G}) .
\end{aligned}
$$

In the same manner we obtain $H_{2 n+1}(\tilde{G})=H_{2 n+1}(G)$. 
Remark. The Jacobi continued fractions of the three power series $G, \bar{G}$ and $\tilde{G}$ defined in Lemma 3.7 are of the following forms:

$$
\begin{aligned}
& G(x)=\mathbf{J}\left[\begin{array}{c}
-b, b,-b, b, \cdots \\
v_{0}, v_{1}, v_{2}, v_{3}, v_{4}, \cdots
\end{array}\right], \\
& \bar{G}(x)=\mathbf{J}\left[\begin{array}{c}
c-b, c+b, c-b, c+b, \cdots \\
v_{0}, v_{1}, v_{2}, v_{3}, v_{4}, \cdots
\end{array}\right], \\
& \tilde{G}(x)=\mathbf{J}\left[\begin{array}{c}
0,0,0,0, \cdots \\
v_{0}, v_{1}, v_{2}, v_{3}, v_{4}, \cdots
\end{array}\right],
\end{aligned}
$$

with the same coefficients $\left(v_{0}, v_{1}, v_{2}, \cdots\right)$.

Acknowledgement. The author would like to thank the referee for his careful reading and his knowledgeable remarks.

\section{References}

[APWW] Allouche, J.-P.; Peyrière, J.; Wen, Z.-X.; Wen, Z.-Y. - Hankel determinants of the Thue-Morse sequence, Ann. Inst. Fourier, Grenoble, 48 (1998), pp. $1-27$.

[B06a] Bacher, Roland. - La suite de Thue-Morse et la catégorie Rec, C. R. Acad. Sci. Paris, Ser. I 342 (2006), pp. 161-164.

[B06b] BACher, Roland. - Recurrence matrices, arxiv.org/abs/math/0601372, 2006, 80 pages.

[Ba09] BARRY, PAUl. - Continued Fractions and Transformations of Integer Sequences, Journal of Integer Sequences, 12 (2009), pp. Article 09.7.6.

[Bu11] Bugeaud, Yann. - On the rational approximation to the Thue-Morse-Mahler numbers, Ann. Inst. Fourier, Grenoble, 61 (2011), pp. 2065-2076.

[BH14] Bugeaud Y.; Han, G.-N. - A combinatorial proof of the non-vanishing of Hankel determinants of the Thue-Morse sequence, Electronic J. Combinatorics, 17 pages, 21(3) (2014), \#P3.26.

[Co13] Coons, Michael. - On the rational approximation of the sum of the reciprocals of the Fermat numbers, The Ramanujan Journal, 30 (2013), pp. 39-65.

[F180] Flajolet, Philippe. - Combinatorial aspects of continued fractions, Discrete Math., 32 (1980), pp. 125-161.

[Go63] Golomb, S.W. - On the sum of the reciprocals of the Fermat numbrs and related irrationalities, Canad. J. Math, 15 (1963), pp. 475-478.

[Gr72] Gros, Luc-AgAthon-Louis. - Théorie du baguenodier par un clerc de notaire lyonnais, Imprimerie d'Aimé Vingtrinier, 1872.

[GX06] Gessel, Ira M.; Xin, Guoce. - The generating function of ternary trees and continued fractions, Electron. J. Combin. 13 (2006), no. 1, Research Paper 53, 48 pp.

[Ha15] Han, Guo-Niu. - Hankel Determinant Calculus for the Thue-Morse and related sequences, J. Number Theory, 147 (2015), pp. 374-395.

[Ha16] Han, GuO-Niu. - Hankel continued fraction and its applications, Adv. in Math., 303 (2016), pp. 295-321.

[HW15] Han, GuO-Niu; Wu, Wen. - Evaluations of the Hankel determinants of a Thue-Morse-like sequence, Int. J. Number Theory, 11 (2015), pp. 1887-1904.

[hKmp] Hinz, Andreas M.; Klavžar, Sandi; Milutinović, Uroš; Petr, Ciril. The tower of Hanoi-myths and maths, Birkhäuser/Springer Basel AG, Basel, 2013 . 
[Kr98] Krattenthaler, Christian. - Advanced determinant calculus, Sém. Lothar. Combin., B42q (1998), 67pp.

[Kr05] Krattenthaler, Christian. - Advanced determinant calculus: A complement, Linear Algebra and its Applications, 411 (2005), pp. 68-166.

[La01] Layman, John W. - The Hankel Transform and Some of its Properties, Journal of Integer Sequences, 4 (2001), pp. Article 01.1.5.

[Pr94] Prodinger, Helmut. - Some information about the binomial transform, Fibonacci Quarterly, 32(5) (1994), pp. 412-415.

[S114] Sloane, N. J. A. - On-line Encyclopedia of Integer Sequences, revision January 24, 2014.

[SS06] Spivey, Michael Z.; Steil, Laura L.. - The $k$-Binomial Transforms and the Hankel Transform, Journal of Integer Sequences, 9 (2006), pp. Article 06.1.1.

[Vi83] VienNOT, X. - Une théorie combinatoire des polynômes orthogonaux généraux, UQAM, Montreal, Quebec, 1983.

[Wa48] WalL, H. S. - Analytic theory of continued fractions, Chelsea publishing company, Bronx, N.Y., 1948.

IRMA, Université de Strasbourg et CNRS

7 rue René Descartes, 67084 Strasbourg, France

guoniu.han@unistra.fr 